\title{
Generalised Residual Stress Depth Profiling at the Nanoscale using Focused Ion Beam Milling
}

\author{
E. Salvati*a, L. Romano-Brandt, M.Z. Mughal', M. Sebastiani ${ }^{b}$, A.M. Korsunsky ${ }^{a}$ \\ ${ }^{a}$ Department of Engineering Science, University of Oxford, Parks Road, Oxford, OX13PJ, \\ United Kingdom \\ ${ }^{b}$ Roma Tré University, Engineering Department, via della Vasca Navale 79, 00146 Rome, Italy

\section{Contents} \\ Keywords..... \\ 1

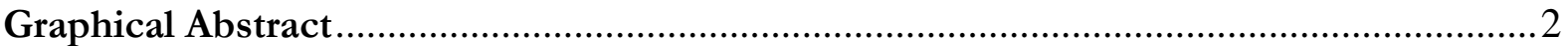

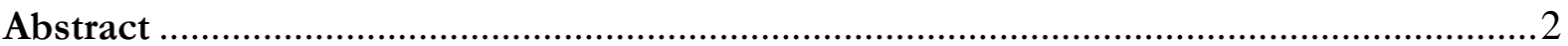

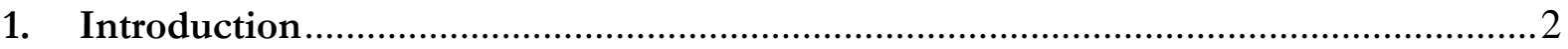

 \\ 3. The generalisation to a Non-Uniform Residual Stress State .....................................5

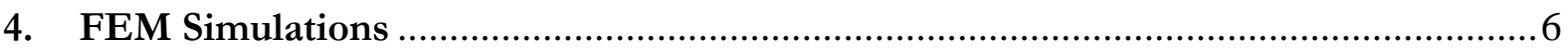

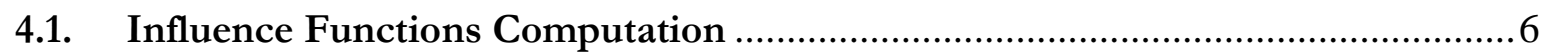 \\ 4.2. Simulated Residual Stress Depth Profiling Process.......................................... 9

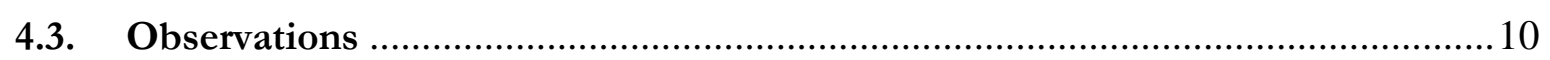

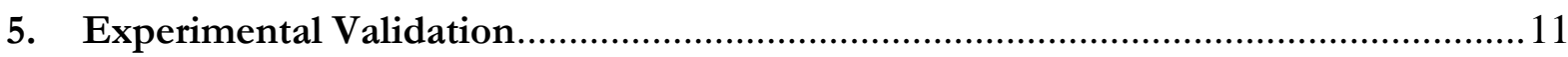

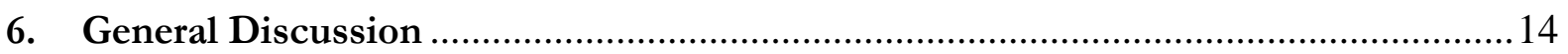

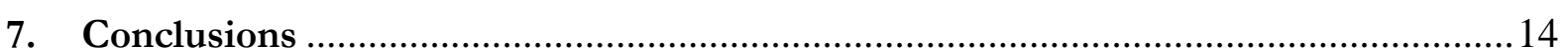

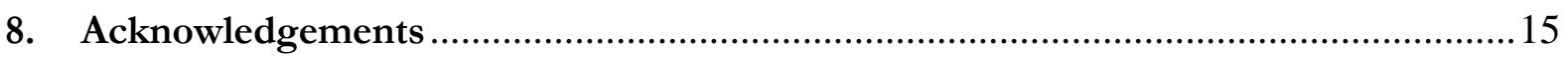

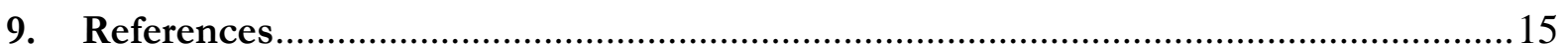

\section{Keywords}

FIB-DIC ring-core; Residual Stress; Eigenstrain; Depth Profiling; non-equi-biaxial

*Corresponding author

E-mail: enrico.salvati@eng.ox.ac.uk; enrico.salvati@outlook.com 


\title{
Graphical Abstract
}

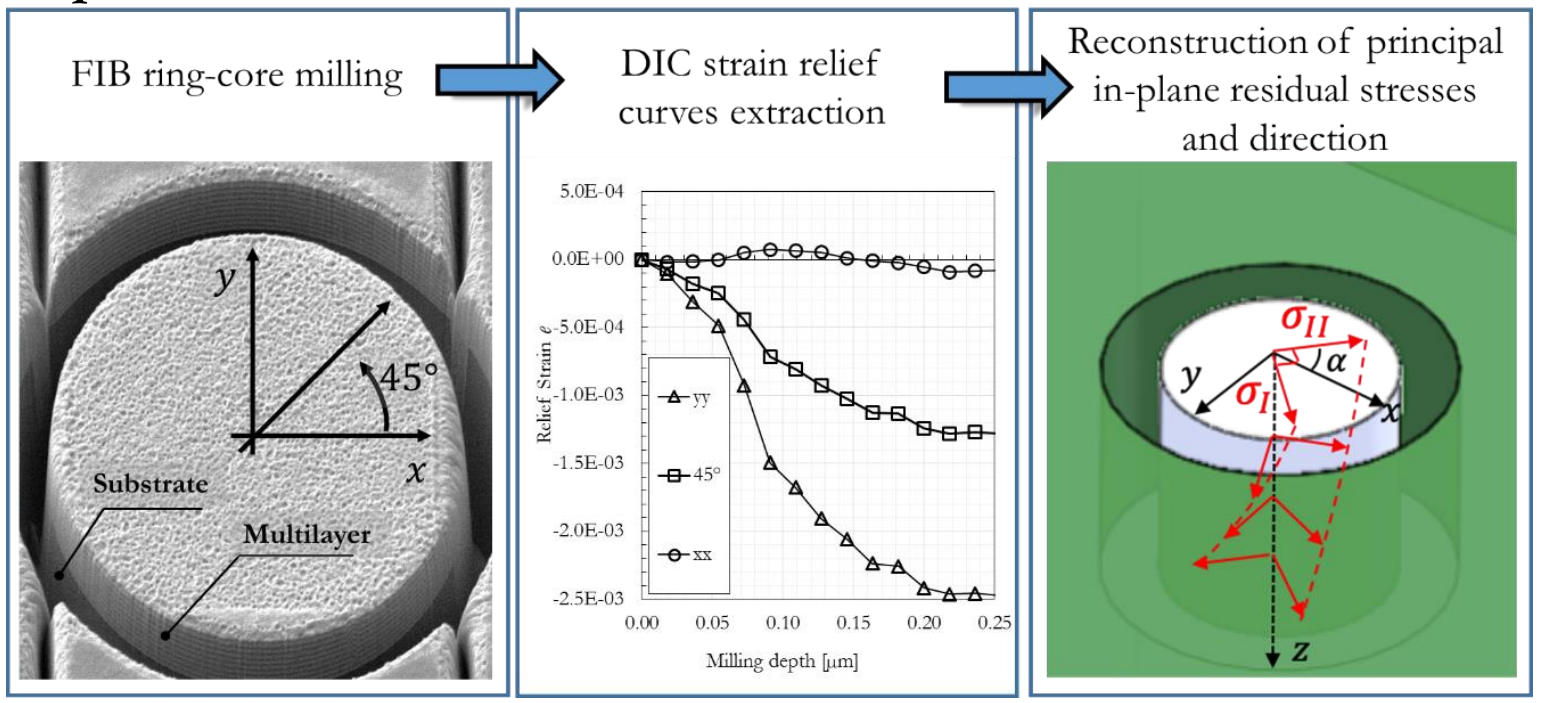

\begin{abstract}
The study of Residual Stress is gaining more and more attention due to its importance in design for structural integrity. At present a lot of emphasis is placed on understanding the origins of mechanical failure that lie at the nano-/micron-scale. This leads to the evident need for evaluating residual stress distributions at increasingly smaller scales, and the search for modern tools capable of accomplishing this task. Prior state-of-the-art methodologies mostly required expensive and time-consuming sample preparation and examination processes to evaluate residual stress, e.g. the study of thin TEM lamellae. The recent advent of Focused Ion Beam methods opened up methods suitable for direct application at sample surface, yet allowing the observation and quantification of stress relief phenomena at the nano-scale. In the last decade, technical aspects of FIB-based method(s) have seen significant development. On the other hand, the calculation framework employed to analyse the experimental outcome remained largely conventional, in most inconvenient for high precision analysis of challenging problems.

In the present paper, the eigenstrain-based method previously presented by the authors for the depthresolved evaluation of equi-biaxial residual stress, is generalised to non-equi-biaxial distributions of residual stress. This extends the applicability of the method to a much wider class of problems. The use of cylindrical ring-core shape in FIB-DIC analysis allows reconstructing the full in-plane residual stress tensor as a function of milling depth. We report formulae for calibrated influence functions that have very broad applicability, and can be used in the overwhelming majority of cases. Their derivation is based on an extensive set of FEM simulations that allowed reliable identification of the limitations of this approach, and highlight the importance of making appropriate selection of ring-core diameter(s). Finally, experimental validation of the method is presented that involves the reconstruction a known non-equibiaxial residual stress depth profile, confirming the validity and reliability of the present approach.
\end{abstract}

\section{Introduction}

Advanced assessment of structural integrity of engineering components relies not only on the intrinsic properties of the material, loading severity and environments, but also on the local material modifications induced by manufacturing processes and treatments. In most of the cases, a conservative approach is taken during the design of new engineering parts, which neglects the presence of many aspects that may concur on the initiation of failures. Inevitably, this strategy leads to the production of non-optimised designs, which impacts the cost and efficiency of the final product. Nonetheless, in some other circumstances, neglecting relevant aspects may even lead to the occurrence of unexpected failures.

One of the features that certainly affects life of mechanical components is the presence of Residual Stress (RS). RS are those stresses that are present in the material in the absence of external loads, and are selfbalanced within the hosting body [1]. Concerning structural integrity, it is well-known how the presence of RS can modify the life performance of a certain component, especially when this is subjected to fatigue loadings [2]. In most cases, the RS field present in a component remains unaltered throughout its life, and 
it is additive to those cyclic stresses induced by external loads during in-service conditions. It is then clear that RS leads fundamentally to a shift of the in-service mean stress compared to the one given by the sole external contribution. As known, modification of the mean stress towards higher values by tensile RS leads to a reduction of the fatigue life, while improvement of performance is experienced in the case of lower mean stresses induced by compressive RS [3, 4].

In general, stress is a scale-dependent concept with a complex interaction with the material's microstructure. The scale-dependent concept can be clearly expressed by introducing the Type I, II and III classification, as first proposed by Pintschovius et. al. [5]. According to this classification, Type I, II and III RS are associated respectively to the macro-scale, micro-scale (or intergranular RS) and nano-scale (or intragranular RS). Moreover, the sum of these RS contribution types gives rise to the actual RS present in a specific location of the considered solid. According to recent experimental and numerical studies, the presence of Type II and III RS may in some cases lead to the variation of RS as high as twice the absolute magnitude of macroscopic RS (Type I) [6, 7]. It is therefore evident how correct evaluation of RS is necessary not only at the macroscopic scale, but also where the fatigue failures originate in the first place, i.e. at the micro- and nano-scale [8].

Families of engineering systems that require particular attention are micro- and nano-scale components, and coated systems. These advanced engineering products are often fabricated using techniques that inherently generate a considerable amount of RS, which may either cause premature failure, or improve performance. For instance, coatings deposited using Physical Vapour Deposition (PVD) processes usually contain RS of compressive nature [9, 10], which are not only effective in combatting crack nucleation but also in improving wear resistance [11]. Another relevant example of micro-scale failure is the crack nucleation at the interface between two different materials, which is a location known to give rise to stress singularity as a consequence of deformation mismatch. The presence of RS can thus be beneficial or detrimental, but is always influential [12].

One of the main challenges in analysing RS lies in the experimental evaluation, particularly at such small scale. While a range of techniques has been developed and validated in the past decades for macroscopic analyses [13-21], the determination of RS at the nano- and micro-scale still has limitations [22]. As far as crystalline materials are concerned, recent studies have shown remarkable resolution of less than $25 \mathrm{~nm}$ achievable for the characterisation of thin films using nano focused Synchrotron based X-ray Powder Diffraction (SXPD) techniques [23, 24]. As well as SXPD, another class of non-destructive techniques employed for RS evaluation at this scale is based on the analysis of Raman scattering [25, 26]. Moreover, the exploitation of Kikuchi patterns acquired through Electron BasckScattered Diffraction (EBSD) technique has recently enabled mapping of RS at the sub-micron resolution [27-30].

However, the major limitations of these techniques are the impossibility of applying them to non-crystalline materials and in some cases the difficulty in determining reference value of RS stress. Furthermore, these methods are generally expensive and time-consuming.

One way to overcome these shortcomings is the exploitation of the strain relief phenomena, occurring in materials containing RS when a cut is performed. As is well recognised, the stress relief taking place by material removal generates distortion of the target part, which can provide valuable information regarding the stress state present before the cut occurred. This principle has been widely exploited at the macro-scale, and several techniques have been established and standardised [13, 20, 31-33]. It is worth mentioning the peculiar characteristic of this family of methods which enable RS evaluation regardless the nature of the material (i.e. amorphous, crystalline, heavily deformed, etc.) and provide absolute quantitative assessment. With the advent of advanced microstructural characterisation tools, such as Scanning Electron Microscopy (SEM) and Focused Ion Beam (FIB), it has become possible in the last decade to transfer the wellestablished strain relief methods from macro- to micro- and nano-scale. The fundamental aspect of these recent advances lies in the use of FIB for the progressive material removal process combined with SEM imaging capability for the step-wise acquisition of the deformed material surface. While at the macro-scale the deformations occurring at the material surface are usually tracked by means of strain gauges, at the smaller scale this task can be accomplished by analysing the acquired images using the Digital Image Correlation (DIC) technique. Strain relief-based techniques at the micro- and nano-scale are generally classified according to the geometrical shape of the milled material, for instance: hole drilling [34], slot cutting [35, 36], cantilever deflection [37], and ring-core milling [38-42].

The ring-core method, also known as the FIB-DIC micro-ring-core method, is attracting a considerable amount of attention thanks to its particular feature of being able to reconstruct the local full in-plane residual stress field by using an automated measurement process $[43,44]$. Particularly in the past few years, 
remarkable advances in this technique were achieved in several key aspects. For example, the quantification of the FIB-induced effect on the evaluated RS [45, 46], the possibility of using local elastic properties for the calculation of RS from residual elastic strain [47] and the capability of reconstructing depth profiles of RS in the case of equi-biaxial RS states [48]. The other major advancement concerns not only the FIB-DIC method, but also other reconstruction techniques based on RS relief phenomena. For the first time, the eigenstrain-based approach was used to overcome the limitations of the conventional technique based on the integral method [32, 49-51]. In fact, the eigenstrain-based calculation framework simplifies the interpretation process considerably, as is does not require invoking Finite Element Method (FEM) for the calculation of sensitivity coefficients for each specific case study.

So far, the application of this novel approach has been limited solely to the case of the residual stress being of equi-biaxial nature. The principal objective of the present paper is the extension of its applicability to any non-equi-biaxial RS state. To accomplish this task, firstly a generalisation of the calculation framework approach is proposed. Subsequently, new FEM simulations are conducted, aimed at evaluating specific influence functions necessary for the reconstruction. Eventually, the method is validated against experimental results. Starting from an equi-biaxial RS state present in a $1 \mu \mathrm{m}$-thick coating, a uniaxial RS state was created by relieving one component via a slit cutting. In this way, a well-known non-uniform RS state was reconstructed by using the newly developed depth profiling FIB-DIC ring-core method. The results of the validation and limitations of the method are widely discussed.

\section{Eigenstrain Depth Profiling Approach}

The formulation recently proposed by Korsunsky et. al [48]- considering solely the case of equi-biaxial RS state - relies on the assumption that the eigenstrain giving rise to RS in the material remains constant throughout the milling process. The term "eigenstrain" identifies the amount of inelastic deformation present in the probed material, which arose during its manufacturing or post-manufacturing processes and which is the cause of the generated residual elastic strain field necessary to keep balance of forces and moments within the body, according to the equilibrium conditions [52, 53]. The assumption of constant eigenstrain is realistic since only negligible variations are induced by the process itself $[45,46,54]$. Therefore, the task of this formulation is the determination of the unknown eigenstrain distribution across the material depth from the measured strain relief evolution and calibrated influence functions. At the depth $h$, the strain relief $e$ can be written as:

$$
e(h / D)=\int_{o}^{\frac{h}{D}} F\left(\frac{Z}{D}\right) \epsilon^{*}(z) \mathrm{d}\left(\frac{Z}{D}\right)
$$

Where $h / D$ is the normalized depth with respect to the core inner diameter $D, z$ is the depth in terms of the eigenstrain $\epsilon^{*}$ and $F\left(\frac{z}{D}\right)$ is the single variable influence function describing the incremental contribution to the surface strain relief from the eigenstrain.

Given the invariance of the eigenstrain during the milling process and therefore not linked to $h / D$, this quantity can be explicitly calculated by differentiating (1) with respect to the variable $h / D$ as follows:

$$
\epsilon^{*}\left(\frac{h}{D}\right)=\frac{\mathrm{d} e(h / D)}{\mathrm{d}(h / D)} / F\left(\frac{h}{D}\right)
$$

At this point, provided that the material substrate is sufficiently thick, and the ratio between the ring-core characteristic length $D$ and the local sample curvature is small enough, the conversion between residual elastic strain $\varepsilon$ and eigenstrain can be simply performed by applying a change in sign as follows:

$$
\varepsilon=-\epsilon^{*}
$$

Eventually, the conversion from residual elastic strain to RS is accomplished by invoking an appropriate version of the Hooke's law, as shown in the next section. 


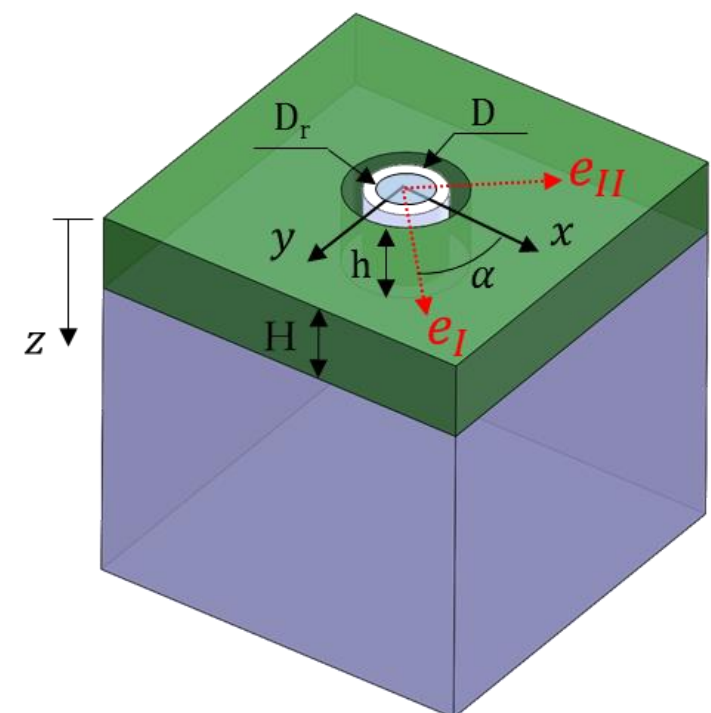

Figure 1. Schematic illustration of a FIB-DIC ring-core milling point. The red arrows indicate the principal in-plane strain relief components.

\section{The generalisation to a Non-Uniform Residual Stress State}

The problem faced in this section deals with the generalisation of the approach described in the previous section. In this case, the sought local RS distribution still varies through the depth but, in the more general scenario, it also varies as a function of the in-plane orientation according to Figure 1. Therefore, at each depth principal RS and directions can vary. In the same way, principal strain relief and directions can also be determined as a function of the depth, and they are named respectively as $e_{I}(h / D), e_{I I}(h / D)$ and $\alpha(h / D)$.

According to the Mohr's circle, the generic expression for in-plane strain relief variation $(e(\varphi))$ is:

$$
e(\varphi)=\frac{e_{I}+e_{I I}}{2}+\frac{e_{I}-e_{I I}}{2} \cos 2(\varphi+\alpha)
$$

Where $\varphi$ is the in-plane generic orientation and $\alpha$ is the angular offset between the original $(\langle x, y\rangle)$ and the principal $(<I, I I>)$ coordinate systems. This formula allows for the determination of the principal strain relief. In general, least square minimization strategy can be adopted to perform this task in case more than three in-plane components are known.

For the sake of simplicity in the implementation of the present calculation, the discrete formulation is considered. Therefore, if three $45^{\circ}$ equally spaced in-plane strain reliefs are monitored throughout the milling, the strain gage rosette theory can be used. These incremental strain reliefs are named as $\Delta e_{x, i .}, \Delta e_{y, i}$. and $\Delta e_{45^{\circ}, i}$, which are respectively associated to the $\mathrm{x}, \mathrm{y}$ and $\alpha=45^{\circ}$ directions, according to Figure 1 . Therefore, at each increment of the milling $i$, the principal strain relief $\left(\Delta e_{1, i}, \Delta e_{2, i}\right)$ and directions $\left(\alpha_{i}\right)$ can be obtained as:

$$
\begin{gathered}
\Delta e_{1, i}=\frac{\left(\Delta e_{x, i}+\Delta e_{y, i}\right)}{2}+\sqrt{\frac{\left(\Delta e_{x, i}-\Delta e_{45^{\circ}, i}\right)^{2}}{2}+\frac{\left(\Delta e_{45^{\circ}, i}-\Delta e_{y, i}\right)^{2}}{2}} \\
\Delta e_{2, i}=\frac{\left(\Delta e_{x, i}+\Delta e_{y, i}\right)}{2}-\sqrt{\frac{\left(\Delta e_{x, i}-\Delta e_{45^{\circ}, i}\right)^{2}}{2}+\frac{\left(\Delta e_{45^{\circ}, i}-\Delta e_{y, i}\right)^{2}}{2}} \\
\alpha_{i}=\frac{1}{2} \tan ^{-1}\left(\frac{\left(\Delta e_{x, i}-\Delta e_{x, i-1}\right)-2\left(\Delta e_{45^{\circ}, i}-\Delta e_{45^{\circ}, i-1}\right)+\left(\Delta e_{y, i}-\Delta e_{y, i-1}\right)}{\left(\Delta e_{x, i}-\Delta e_{x, i-1}\right)-\left(\Delta e_{y, i}-\Delta e_{y, i-1}\right)}\right)
\end{gathered}
$$

Once known the incremental strain relief tensor is defined in the principal directions, a decomposition can be conducted, which separates the hydrostatic component from the deviatoric one. In this fashion, two strain tensors are associated to the isotropic deformation (volume change, $\overline{\overline{\Delta e_{H_{l}}}}$ ) the first and the second 
to those components that induce shape change (pure shear, $\overline{\overline{\Delta e_{D_{l}}}}$ ). In general, at each milling step $i$, it is always possible to define the total tensor as a sum of these two:

$$
\overline{\overline{\Delta e_{l}}}=\overline{\overline{\Delta e_{H_{l}}}}+\overline{\overline{\Delta e_{D_{l}}}}
$$

Also, in its explicit form, showing the tensorial elements:

In which:

$$
\left(\begin{array}{cc}
\Delta e_{1, i} & 0 \\
0 & \Delta e_{2, i}
\end{array}\right)=\left(\begin{array}{cc}
\Delta e_{H, i} & 0 \\
0 & \Delta e_{H, i}
\end{array}\right)+\left(\begin{array}{cc}
\Delta e_{D, i} & 0 \\
0 & -\Delta e_{D, i}
\end{array}\right)
$$

$$
\Delta e_{H, i}=\frac{\left(\Delta e_{1, i}+\Delta e_{2, i}\right)}{2} ; \quad \Delta e_{D_{1}, i}=\frac{\left(\Delta e_{1, i}-\Delta e_{2, i}\right)}{2}
$$

This is allowed since the formulated matrix is a tensor, and therefore its first invariant of the deviatoric tensor must be zero, therefore its trace:

$$
\operatorname{Tr} \Delta e_{D}=\Delta e_{D_{11}, i}+\Delta e_{D_{22}, i}=0
$$

At this point it is possible to extend the method originally applied solely to the equi-biaxial strain case to the case of arbitrary in-plane strain distribution. Before doing that, a normalised finite milling depth needs to be defined as follows:

$$
\frac{h_{i}^{*}}{D}=\frac{1}{D}\left(\frac{h_{i}+h_{i-1}}{2}\right)
$$

In this manner, a more appropriate depth is considered when the influence functions are computed. Indeed, at each milling step $i$ the depth accounted lies exactly in the middle of the actual step $i$ and the previous $i-$ 1.

According to the original depth profiling method [48], the actual residual elastic strain present at the normalised depth $\frac{h_{i}^{*}}{D}$ for the equibiaxial case can be calculated as the incremental strain relief divided by the corresponding value of the influence function $\mathrm{F}_{H, i}$, changed in sign to pass from eigenstrain to residual elastic strain, as shown in (2) and (14). For this particular case, this incremental strain relief corresponds to the hydrostatic (or more precisely, equi-biaxial) part of the strain state. However, in the general case, a deviatoric part of the strain state may exist and thus needs to be evaluated using the appropriate influence function, denoted $\mathrm{F}_{D, i}$. Therefore, the principal elastic residual strains can then be obtained as:

$$
\left(\begin{array}{l}
\varepsilon_{1, i} \\
\varepsilon_{2, i}
\end{array}\right)=-\frac{1}{\mathrm{~F}_{H, i}}\left(\begin{array}{c}
\Delta e_{H, i} \\
\Delta e_{H, i}
\end{array}\right)-\frac{1}{\mathrm{~F}_{D, i}}\left(\begin{array}{c}
\Delta e_{D_{1}, i} \\
-\Delta e_{D_{1}, i}
\end{array}\right)
$$

where $\mathrm{F}_{H}$ and $\mathrm{F}_{D}$ are the influence functions respectively for the hydrostatic and deviatoric parts. These functions are computed by means of FEM analyses and this calculation is shown in the next section of the paper. Note that when the strain state is purely equi-biaxial, then only the hydrostatic term is relevant. In the other extreme case, when a pure in-plane shear strain state is present, then the deviatoric term is the only one that is relevant. In the general circumstance, both the equi-biaxial (hydrostatic) and pure shear (deviatoric) influence functions must be applied.

Eventually, the principal residual stress state can be found assuming that the out-of-plane residual stress components are close to zero within the probed volume. Therefore, the residual stress tensor in the principal directions can be evaluated by an appropriate form of Hooke's law as follows:

$$
\left[\begin{array}{l}
\sigma_{I} \\
\sigma_{I I}
\end{array}\right]=\frac{E}{\left(1-v^{2}\right)}\left[\begin{array}{ll}
1 & v \\
v & 1
\end{array}\right]\left[\begin{array}{c}
\varepsilon_{I} \\
\varepsilon_{I I}
\end{array}\right]
$$

However, the calculation shown in (14) can only be applied when the material elastic properties are assumed isotropic. Whereas the local elastic properties show anisotropic behaviour, the calculation of RS can be performed using the procedure shown by Salvati et al. [47]. This procedure allows for the evaluation of RS even if the local crystal orientation is unknown, by accounting uncertainties generated upon it.

\section{FEM Simulations}

\subsection{Influence Functions Computation}

To accomplish this task in an accurate and computationally inexpensive way, a 3D FEM model was built within the COMSOL environment. In order to reduce the computational cost further, only one quarter of the model was simulated, by exploiting the geometry's symmetry properties as shown in Figure 2(a). As it is possible to observe from this Figure, gradual mesh refinement was produced in the proximity of regions where high gradients of stress and strain are expected, therefore at the periphery of the machined pillar 
outer surface. Moreover, mesh refinement was done at the shallowest layer of the ring core, where the most significant strain relief takes place. However, a mesh sensitivity analysis was carried out, aimed at searching for the best compromise between accuracy and computational speed. A further check of result accuracy was done by comparing the sensitivity functions obtained in a previous publication, in which an axisymmetric FEM model was employed with a higher degree of mesh refinement, in the sole case of equibiaxial through-depth uniform eigenstrain distribution [48].

A time-resolved calculation was employed for the simulation of the whole milling process. Material removal was simulated by setting the Young's modulus of the elements being removed to a near null value.

Constant through-thickness eigenstrain distributions were prescribed within a shallow layer of thickness $H$, as depicted in Figure 2(b). To extract the two sought sensitivity functions, both an in-plane isotropic and an orthotropic (pure in-plane shear) distributions of eigenstrain were prescribed, respectively to evaluate $\mathrm{F}_{H}$ and $\mathrm{F}_{D}$. According to Figure 2(b), the simulation of $\mathrm{F}_{H}$ was performed imposing $\epsilon_{x}^{*}=\epsilon_{y}^{*}$ and, on the other hand, $\mathrm{F}_{D}$ was found by assuming $\epsilon_{x}^{*}=-\epsilon_{y}^{*}$ which resembles a pure in-plane shear state.


Figure 2. Model illustration. (a) Meshed 3D domain and highlight of the material removed region, (b) Eigenstrain components prescription example

The strain relief is calculated over an effective area of the produced pillar top surface as also shown in [48], identified as $A_{e}=\frac{\pi}{4} D_{r}{ }^{2}$. Where $D_{r}$ is the reduced diameter, defined through an area reduction factor $\delta=$ $D_{r} / D$. Such reduction is employed in this geometry to avoid possible artefacts from the pillar's edges. These results are reported in Figure 3(a) as a function of the normalized depth $h / D$, of the area reduction factor and either equi-biaxial or pure shear eigenstrain distribution.

With the purpose of illustrating the main differences regarding sensitivity functions, the relief curves illustrated in Figure 3(a) were fitted using quadratic-exponential fitting functions reported in [48], and subsequently differentiated to obtain the actual sensitivity functions, reported in Figure 3(b). 

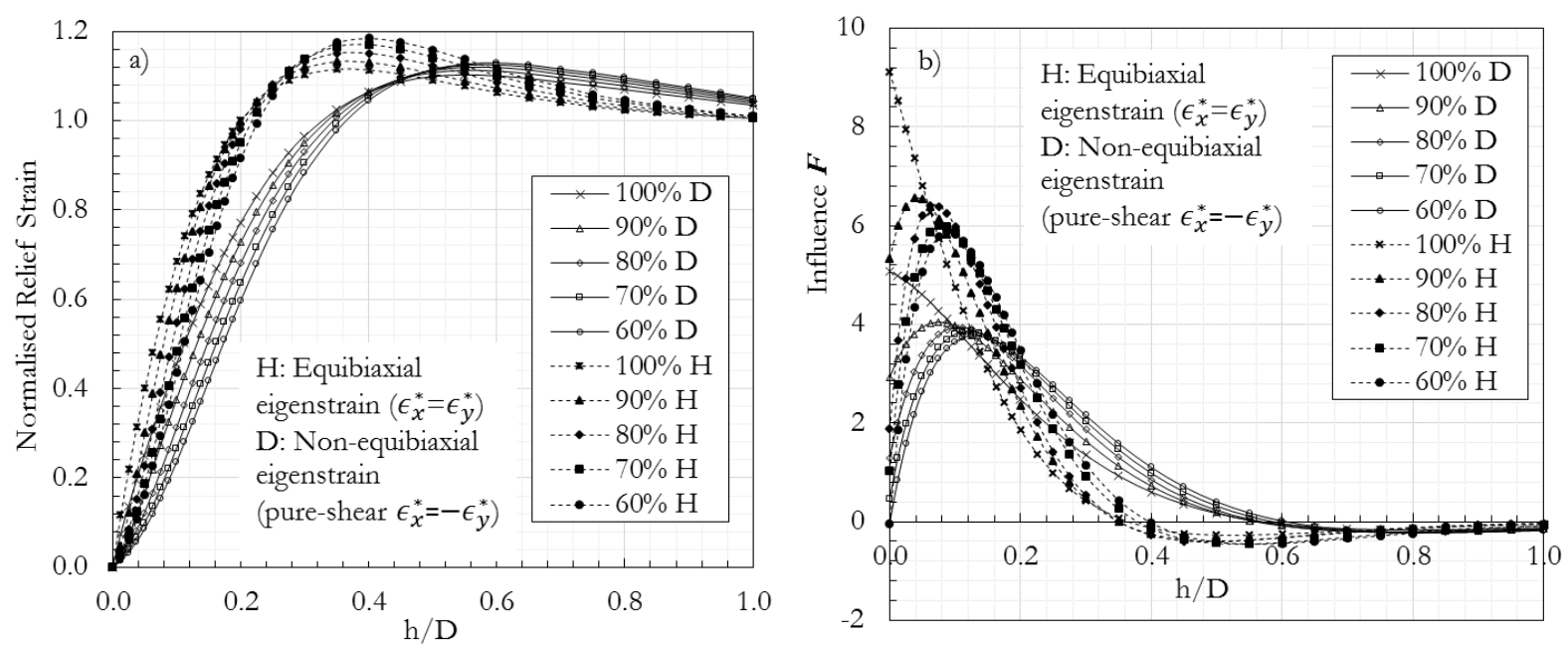

Figure 3. FEM results at several DIC area reductions. (a) Strain relief curves. (b) Sensitivity functions.

Nevertheless, after detailed simulations of the entire milling process in non-constant through-thickness eigenstrain distribution, shown in the next section, it has become evident the necessity of refining the accuracy of these functions in the range $0<\frac{h}{D} \leq 0.2$, and neglecting their behavior for $\frac{h}{D}>0.2$. To improve the analytical representation of these functions, in this paper $3^{\text {rd }}$ order exponential-polynomial functions are utilised to describe the FEM trend within the range of interest. The function used to describe the strain relief is:

$$
f\left(\frac{h}{D}\right)=1-\exp ^{-a\left(\frac{h}{D}\right)}\left[1+b\left(\frac{h}{D}\right)-c\left(\frac{h}{D}\right)^{2}+d\left(\frac{h}{D}\right)^{3}\right]
$$

Therefore, the sensitivity functions $F\left(\frac{h}{D}\right)$ can be easily found by differentiating it with respect to $\frac{h}{D}$. Hence:

$$
F\left(\frac{h}{D}\right)=\exp ^{-a\left(\frac{h}{D}\right)}\left[a-b+(2 c+a b)\left(\frac{h}{D}\right)-(3 d+a c)\left(\frac{h}{D}\right)^{2}+a d\left(\frac{h}{D}\right)^{3}\right]
$$

\begin{tabular}{|c|c|c|c|c|c|c|c|c|c|c|}
\hline \multirow[b]{2}{*}{ Coeff. $\delta$} & \multicolumn{5}{|c|}{$F_{H}$} & \multicolumn{5}{|c|}{$\boldsymbol{F}_{D}$} \\
\hline & 100 & 90 & 80 & 70 & 60 & 100 & 90 & 80 & 70 & 60 \\
\hline$a$ & $1.882 \mathrm{E}+00$ & $1.077 \mathrm{E}+01$ & $9.633 \mathrm{E}+00$ & $1.126 \mathrm{E}+01$ & $1.066 \mathrm{E}+01$ & $9.247 \mathrm{E}+00$ & $7.044 \mathrm{E}+00$ & $6.208 \mathrm{E}+00$ & $2.371 \mathrm{E}+00$ & 9.188E-01 \\
\hline$b$ & $-7.388 \mathrm{E}+00$ & $6.897 \mathrm{E}+00$ & $7.975 \mathrm{E}+00$ & $9.872 \mathrm{E}+00$ & $9.349 \mathrm{E}+00$ & $3.212 \mathrm{E}+00$ & $4.746 \mathrm{E}+00$ & $5.026 \mathrm{E}+00$ & $1.435 \mathrm{E}+00$ & $9.527 \mathrm{E}-02$ \\
\hline$c$ & $-1.174 \mathrm{E}+01$ & $5.858 \mathrm{E}+01$ & $6.099 \mathrm{E}+01$ & $1.663 \mathrm{E}+01$ & $5.446 \mathrm{E}+00$ & $-1.465 \mathrm{E}+01$ & $2.084 \mathrm{E}+01$ & $2.226 \mathrm{E}+01$ & $2.540 \mathrm{E}+01$ & $2.105 \mathrm{E}+01$ \\
\hline$d$ & $8.223 \mathrm{E}-01$ & $1.003 \mathrm{E}+00$ & $-6.146 \mathrm{E}-01$ & $-2.265 \mathrm{E}+02$ & $-2.406 \mathrm{E}+02$ & $-9.571 \mathrm{E}+01$ & 1.158E-01 & 6.862E-02 & $3.982 \mathrm{E}+01$ & $3.883 \mathrm{E}+01$ \\
\hline
\end{tabular}

After curve fitting, the resulting constants were found and are summarized in the following table:

Results of the refined fittings at the range of interest are shown in the Figure 4. 

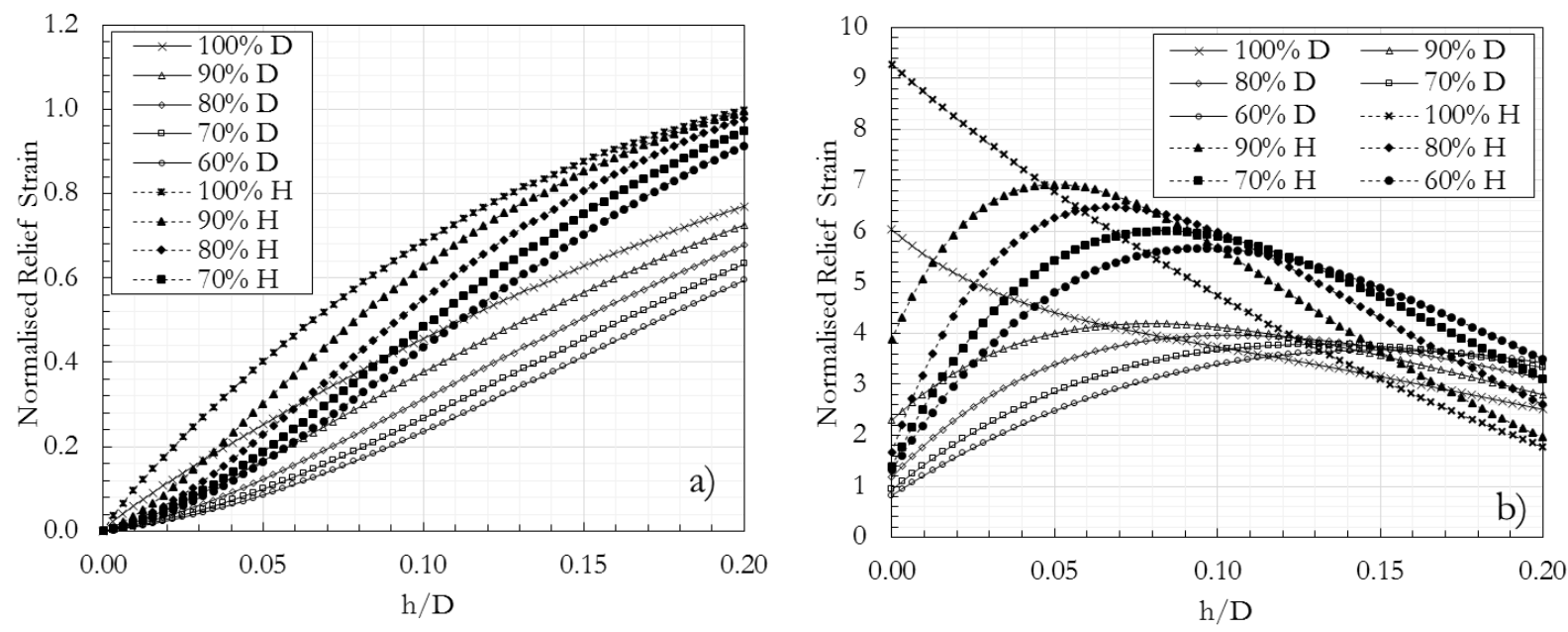

Figure 4. Sensitivity analysis (a) Strain relief curves from FEM. (b) Influence functions.

\subsection{Simulated Residual Stress Depth Profiling Process}

In this section, simulations of the ring-core milling process and subsequent residual elastic strain reconstruction are performed. In order to assess the accuracy of the proposed method, several case-studies are shown, in which relevant strain gradients and non-uniform residual elastic strain distribution were prescribed within the FEM model. In order to test the performance of the proposed method it is necessary to define the severity of residual elastic strain at which the reconstruction fails evaluating the actual profile. Since in this work - for the sake of simplicity - the residual elastic strain profiles are defined as linear, it is possible to define a linear gradient of strain as:

$$
\varepsilon^{\prime}=\frac{\Delta \varepsilon}{(\Delta h / D)}
$$

Where $\Delta \varepsilon$ is the variation of strain within a certain normalised depth interval, $\Delta h / D$.

Three case-studies are reported in this section, considering an area reduction factor $\delta=80$. Firstly, an equi-biaxial linearly decreasing compressive residual elastic strain was prescribed in the model, up to the normalised depth of 1 , as shown in Figure 5(b). In this case, the gradient of strain was set to $5 \%$. Such distribution of residual elastic strains gave rise to the relief curve depicted in Figure 5(a).

Alongside the prescribed profile, Figure 5(b) shows the reconstructed profile, using markers.
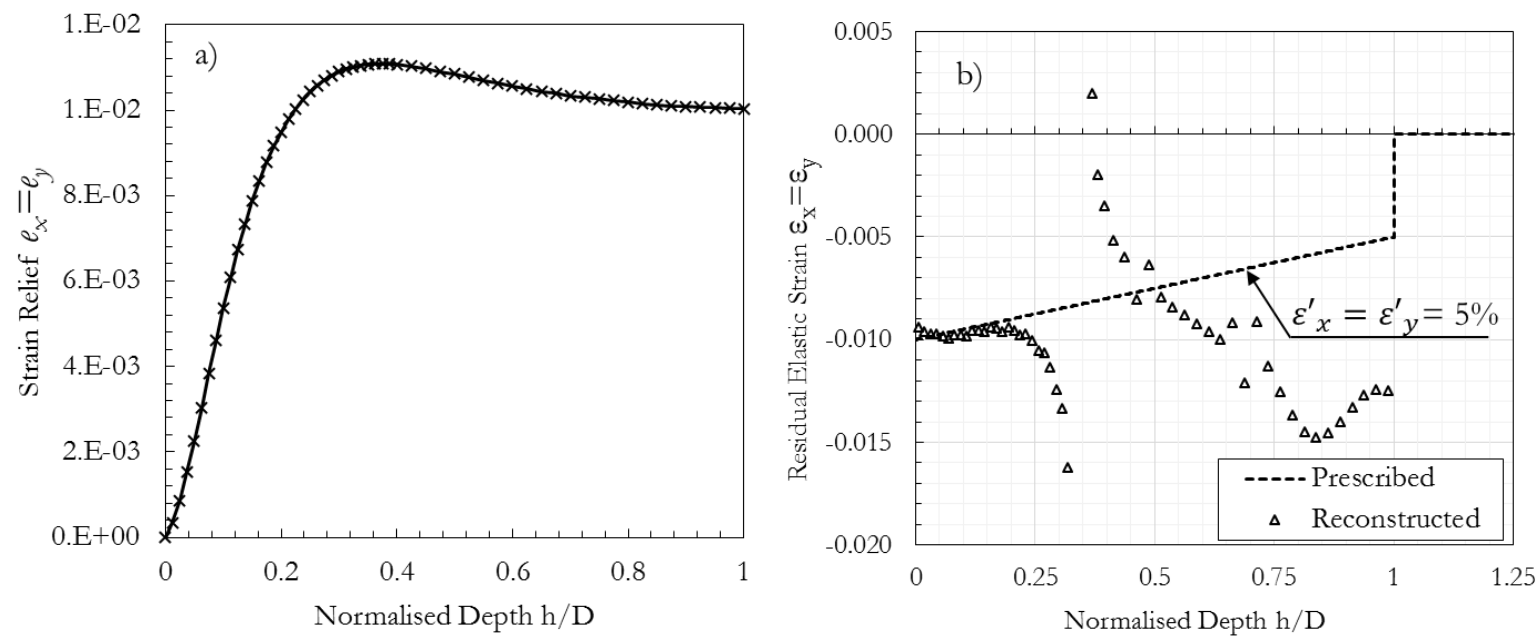

Figure 5. Arbitrary equi-biaxial residual elastic strain profile $\varepsilon_{x}^{\prime}=\varepsilon^{\prime}{ }_{y}=5 \%$. (a) Strain relief curve (b) Comparison

The second case-study was focused on the analysis of a non-equi-biaxial residual elastic strain distribution. Even in this instance, the depth profiles were assumed linear and the gradients of strain were assumed 1.5\% and $0.5 \%$ respectively for the $x$ and $y$ principal directions. Conversely from the previous case-study where 
the strain relief was accounted up to the depth of $h / D=1$, in this example the reconstruction was conducted up to the normalised depth of $h / D=0.2$ and reported in Figure 6. This choice was dictated by the fact that the method fails to reconstruct the residual elastic strain for deeper positions, as demonstrated in Figure 5(b). Further comments on this point are given in the next section.


Figure 6. Arbitrary non-equi-biaxial residual elastic strain profile $\varepsilon_{x}^{\prime}=0.5 \%, \varepsilon_{y}^{\prime}=1.5 \%$. (a) Strain relief curves (b) Comparison

Eventually, the last case-study attempts at reconstructing high linear gradients of non-equi-biaxial residual elastic strain depth profiles; in this example $\varepsilon^{\prime}$ was chosen to be $13.5 \%$, as shown in Figure 7.
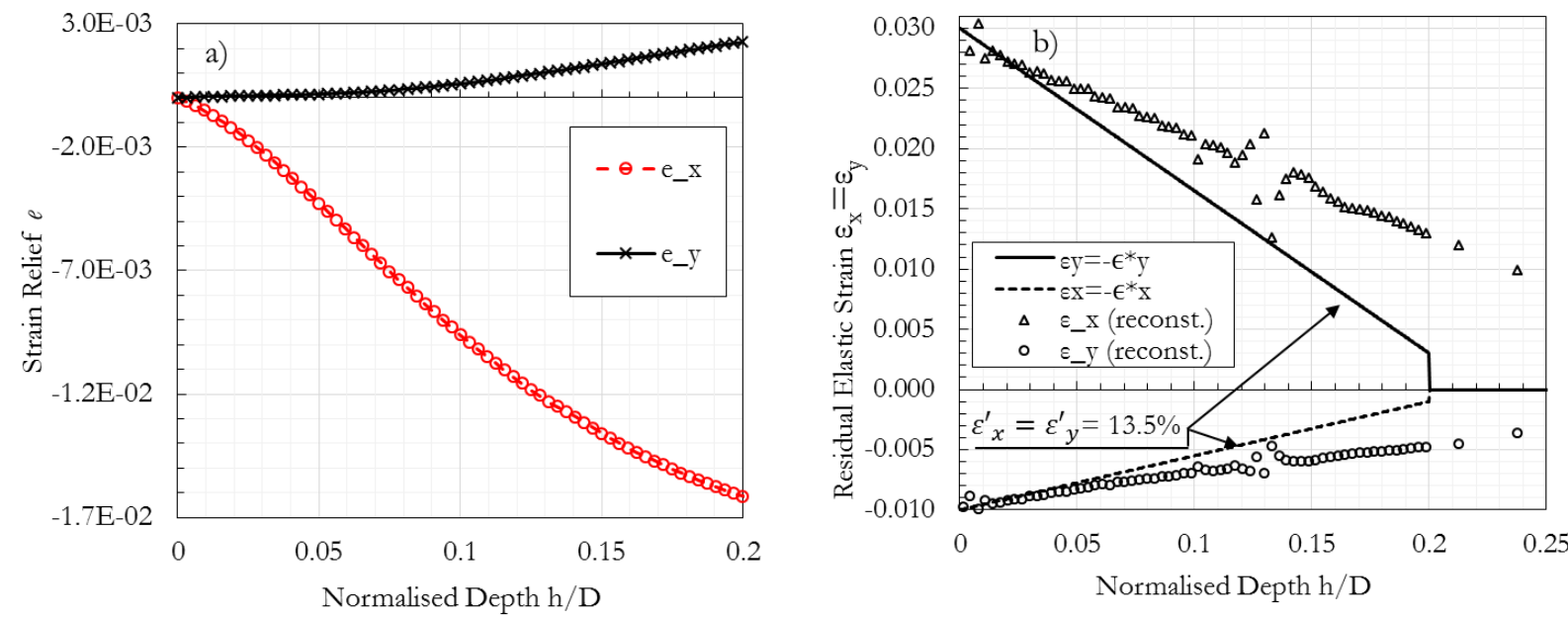

Figure 7. Arbitrary non-equi-biaxial residual elastic strain profile $\varepsilon^{\prime}{ }_{x}=\varepsilon^{\prime}{ }_{y}=13.5 \%$. (a) Strain relief curves (b) Comparison

\subsection{Observations}

The FEM analyses shown in this section shed some important light on the capability of the presented method. First of all, it has been demonstrated how the accuracy of the method decays when the analysed strain relief is associated to depths larger than $h / D=0.2$. In fact, in Figure 5 (b) shows that for a linear strain gradient of $5 \%$, an excellent accuracy of reconstruction is obtained. This particular behaviour of the method was already known and reported in a previous publication of the authors [48]. In addition to that, it is now evident the reason why this effect appears. As visible in Figure $5(\mathrm{~b})$ at the depth of $h / D \approx 0.3$, a singularity is experienced. This is because the associated sensitivity function $\left(F_{H}\right)$ assumes null value at this depth. Thereby, according to equation (14), the division operation leads to infinite value of residual elastic strain, which is not realistic. Evidently, as the considered depth approached such singularity, the reconstructed residual elastic strain field is affected and gradually fails to capture the correct values.

Nevertheless, by discarding the reconstruction at positions $h / D>0.2$, the method correctly captures the actual residual elastic strain. Indeed, it can be concluded that if the gradient of strain $\varepsilon^{\prime}$ is $5 \%$ or less, the 
relative discrepancy from the prescribed values is less than $5 \%$, neglecting very shallow depths. It is surely worth discussing the fact that at the shallowest depths the method highlights some divergences, which are imputed to a geometrical effect induced by the trench. Such an effect was already known [48], and for this reason, the range $0>h / D>0.01$ is usually neglected in the reconstruction.

According to Figure 6, the method also excellently captures the residual elastic strain profile when the strain in non-equi-biaxial. In this case the relative error experienced was again less than $5 \%$. This result demonstrates the correctness of the proposed approach to reconstruct arbitrary non-equibiaxial RS fields, provided that adequate ring-core diameter is selected for the specific application; i.e. the diameter to be used is $D=h_{\max } / 0.2$, where $h_{\max }$ is the maximum depth at which the reconstruction needs to be performed with a single ring core.

On the other hand, it was observed how the method fails to reconstruct high gradients of residual elastic strains, as shown in Figure 7(b). In this instance, an acceptable result is only limited within the range $0.01>$ $h / D>0.03$, after which the deviation rises to $100 \%$, making the method unreliable. A systematic error was found to be present, which modified the slope of the reconstructed profile. Nonetheless, the limitation of the method highlighted by this case-study $\left(\varepsilon^{\prime}=13.5 \%\right)$ can be overcome by selecting a suitable ring core diameter, and this can be promptly seen from equation (17). In fact, by employing different diameter of the ring-core, $\varepsilon^{\prime}$ changes too. In a practical case, smaller ring-core diameter is reflected into a reduction the magnitude of $\varepsilon^{\prime}$, making the latter small enough to reliably exploit the method.

\section{Experimental Validation}

In this section, experimental validation of the proposed calculation methodology is shown. Validation of the method must be accomplished by evaluating a well-known residual stress depth profile. To achieve this goal, for the present experiment we employed a $1 \mu \mathrm{m}$ thick multilayer coating of ten $\mathrm{Cu} / \mathrm{W}$ bilayers exhibiting an equi-biaxial residual stress state. The same sample has been extensively probed and presented in a recent publication by the authors, where residual stress was assessed by three independent experimental techniques, namely: FIB-DIC ring-core, XRD and micro-cantilever deflection [55]. With the purpose of probing a non-equi-biaxial residual stress distribution across the coating thickness, two parallel $10 \mu \mathrm{m}$ spaced slits were machined, making sure that the cut was deep enough to completely relieve the residual stress component in direction perpendicular to the slits extent, in the region confined by the two cuts; an example of the machined slits and ring-core is shown in Figure 10(b). Four equi-spaced ring-cores having diameter $D$ of $10 \mu \mathrm{m}$ were machined. Spacing between them was set to $5 \mathrm{D}$ to minimise mutual disturbance, according to the simulation shown in [44]. Such diameter was selected to allow full reconstruction of the 1 $\mu \mathrm{m}$ thick coating and a further micron lying within the Si substrate. It is worth remembering that good accuracy is obtained at normalised depths of $\mathrm{h} / \mathrm{D}<0.2$, therefore in this case this range covers the $2 \mu \mathrm{m}$ thick layer probed. The pillar area reduction factor $\delta$ was selected equal to 80 , to avoid edge effects and guarantee good statistics of the DIC tracked points. Elastic properties of the material were assumed to be isotropic. The elastic modulus and Poisson's ratio of the $\mathrm{Cu} / \mathrm{W}$ multilayer were respectively assumed equal to $220 \mathrm{GPa}$ and 0.35 [56]. While for the substrate these values were $160 \mathrm{GPa}$ and 0.278 , respectively for the elastic modulus and Poisson's ratio.

Analysis of the deformation taking place on the surface of the machined pillar was conducted by processing images acquired via SEM using a MATLAB-based DIC software [57]. Since the full in-plane strain relief tensor was sought, according to Figure 8, three directions were analysed, namely 0, 45 and 90 degrees from the reference $\mathrm{x}$-axis. The same figure reports a summary of the evaluated strain relief profiles. 



Figure 8. Strain relief profiles along different directions. (a) 0 degrees (b) 45 degrees (c) 90 degrees. The inner ring-core diameter shown in the inset is $10 \mu \mathrm{m}$. (d) SEM overview image of the slits and ring-core.

The calculation procedure shown earlier was followed for each triplet of strain relief curves shown in Figure 8. This allowed for the reconstruction of four RS depth profiles for each principal direction, shown in Figure 9 by the continuous black lines - the calculation solved equation (14) invoking appropriate elastic properties along the sample depth, according to characteristic material composition. The averaged profiles, respectively for the first and the second principal RS, are also reported by using a red thick continuous line, along with the confidence band confined by two red dotted lines, indicating $95 \%$ of confidence. It is worth mentioning that such confidence band was only defined by accounting the scatter from each measurement point. In fact, some sources of errors were deliberately neglected in this case-study. For instance, some of the errors that in some cases may be relevant are due to the DIC tracking or due to uncertainty in measurements of the correct depth at each milling step; this aspect will be thoroughly faced in a separate work. 

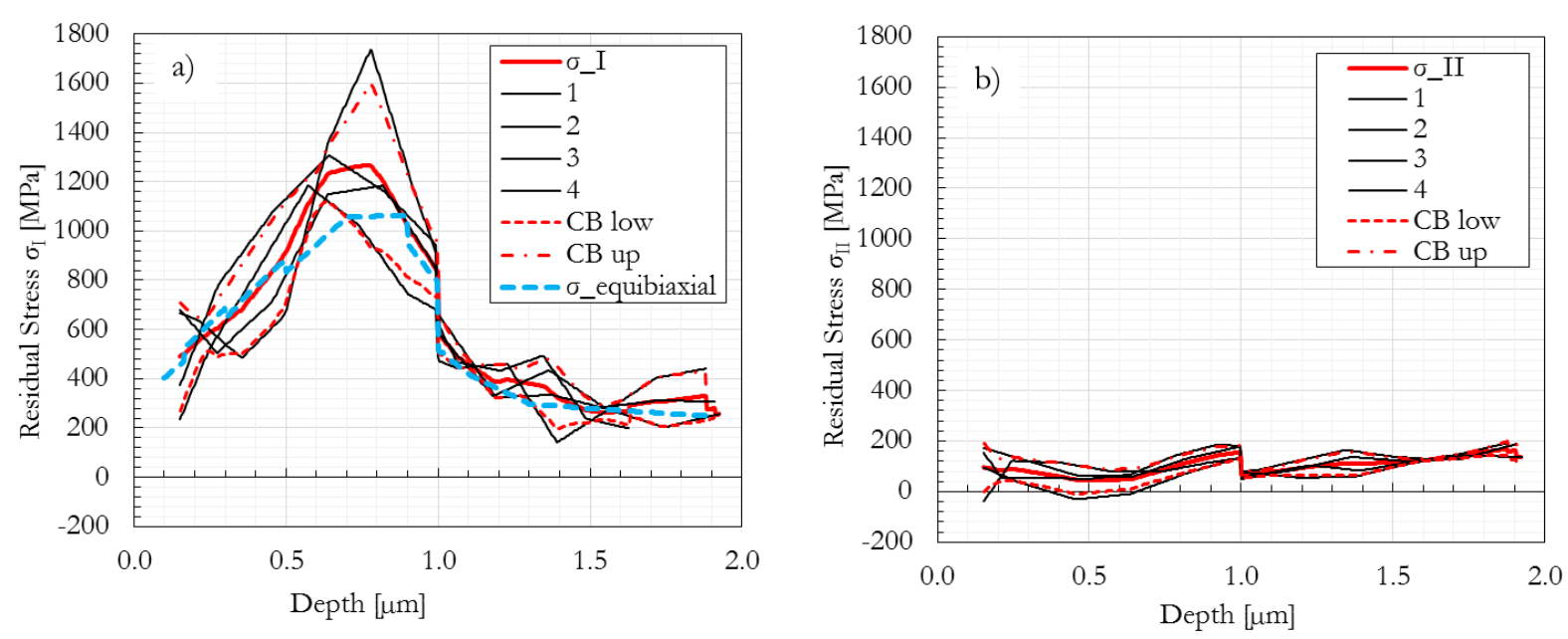

Figure 9. Depth resolved Residual Stress profiles. (a) First principal. (b) Second principal.

To fully characterise the local RS field, the principal directions are required as well. With this purpose, by invoking equation (7), the rotation angle $\alpha$ which identifies the principal stress coordinate system with respect to the global coordinate system is calculated through the thickness. Graphical illustration of coordinate systems onto an actual ring-core pillar is depicted in Figure 10(b). According to this illustration, the angle $\alpha$ is plotted as a function of the depth in Figure 10(a). Again, continuous black lines refer to individual ring-cores, while the red lines are the averaged values and associated error band limits.
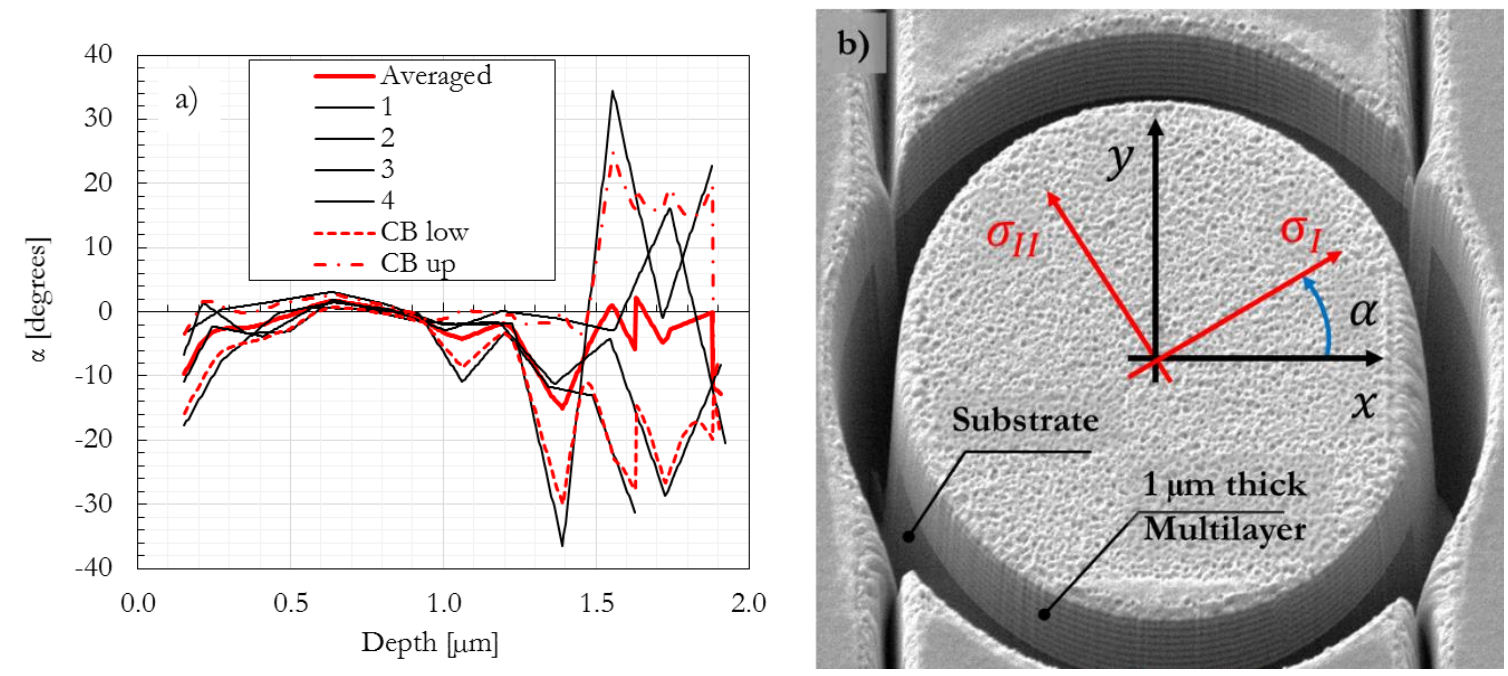

Figure 10 Principal directions. (a) depth resolved principal direction. (b) Illustration.

Analysis of principal direction orientation showed a substantial alignment of these with the global coordinate system defined prior the ring-core milling, highlighted by the near zero angle $\alpha$ within the $1 \mu \mathrm{m}$ multilayer (Figure 10(a)). It is worth observing that some unexpected rotation of the principal directions was experienced both at the shallowest regions and at depths higher than $1.25 \mu \mathrm{m}$, although on an average not exceeding 14 degrees. This effect can be imputed to the low magnitude of RS within these regions, as it can be seen from Figure 9. In fact, such low magnitude is comparable with the expected sum of the intrinsic errors carried by this method. When the difference between the two principal RS in terms of magnitude is comparable to the reconstruction scatter, it becomes difficult to identify principal directions accurately. Nevertheless, the reconstruction of principal directions can be considered satisfactory.

Since in this case the principal directions are generally aligned with the global coordinate system, the first principal RS $\sigma_{I}$ can be directly compared with the equi-biaxial RS state $\sigma_{e q}$. analysed in a previous publication [55]. The RS depth profile results are shown in Figure 9(a) clearly show excellent agreement between the two. The machining of two parallel slits allowed for relieving the sole RS component 
perpendicular to those slits, therefore the corresponding perpendicular component was supposed to remain unchanged, and indeed this happened. For both the reconstruction tensile residual stress of $\sim 500 \mathrm{MPa}$ was detected at a depth of $0.15 \mu \mathrm{m}$, followed by a gradient which leads to a maximum value of $\sim 1250 \mathrm{MPa}$ and a decay down to $\sim 300 \mathrm{MPa}$ when the substrate is reached. Note that at a depth of $1 \mu \mathrm{m}$, corresponding to the multilayer/substrate interface, a discontinuity is present. This is solely due to the different elastic properties of the two materials, although a smooth transition is expected. Also, in this case, the gradient of residual elastic strain defined in equation (17) was quantified to be $\sim 7 \%$, therefore close to the $5 \%$ simulation shown earlier which demonstrated excellent quality of the reconstruction.

As far as the second principal RS component is concerned (Figure 9(b)), some relevant observation can be drawn. The reconstruction overall is satisfactory as the mean value is $100 \mathrm{MPa}$, meaning that the stress along the $x$-direction took place, although not precisely to a null value. The fact that deeper positions such as $h>0.7 \mu \mathrm{m}$ show a gradual increase of the second principal RS magnitude hints that a mild effect of the out-of-plane component of RS can be present. While the assumption of plane-stress is certainly valid at the sample free surface, this becomes less and less valid as the probed material is at deeper positions. Nevertheless, the influence of the out-of-plane component in this case-study can be considered negligible given its limited effect.

\section{General Discussion}

The outcomes of the FEM and experimental analysis demonstrated the correctness of the generalised reconstruction method based on eigenstrain.

On the one hand, the exhaustive FEM simulations allowed for the identification of main limitations of the method and which precautions need to be taken to overcome these effectively. Two main aspects need to be assessed before and after the evaluation procedure to correctly evaluate the RS in coating systems. Of paramount importance is the diameter of the ring-core that must be selected considering the thickness of the probed material in the first instance. Also, its appropriateness needs to be checked after the reconstruction of the RS profile to make sure the measured gradient of residual elastic strain or RS is not too high. It is worth reminding that the method cannot be applied in cases where the thickness of the material is comparable with the depth extent of the eigenstrain within the probed sample. In fact, in this particular case, the assumption of eigenstrain being the same but changed in sign of the residual elastic strain decays. For instance, the introduction of a coating or a surface treatment on a sample can be thought as an inelastic deformation taking place within this affected region, namely eigenstrain. In order to reestablish balance of internal forces in the sample, residual elastic strain must originate. If the thickness of the sample is much larger than this affected layer where the eigenstrain is present, then the residual elastic strain will predominantly arise in correspondence of the eigenstrain location, as outside this region the balance of forces and moments will be ensured by a very low residual elastic strain. Conversely, if the thickness of the treated sample is comparable to the induced eigenstrain depth, then the absolute magnitude of residual elastic strain field outside the eigenstrain field will be significant as limited depth is available to accommodate the balancing forces required. Nevertheless, in case this issue arises, it can be surmounted by simulating bespoken influence functions for the particular scenario or simulating the residual elastic strain from the extracted eigenstrain profile and geometry of the sample. This particular case will be elucidated in a future work.

On another hand, experimental evidence was provided to validate the proposed method. Undeniably, the processed experimental results demonstrated the capability of the method of resolving non-equi-biaxial depth profiles of RS at (sub)micron resolution which is remarkable.

\section{Conclusions}

A generalised method based on eigenstrain has been shown to overcome the main limitations of classic integral methods. First, the sole influence functions computed and presented in this paper can be applied in the overwhelming majority of applications dealing with coatings and treated surface. Therefore, incurring in time consuming FEM simulations for each specific case-study is no longer necessary. Mainly for the FIBDIC ring-core method, it was shown that the application of the proposed methodology could achieve remarkable performance in terms of depth resolution (better than $100 \mathrm{~nm}$ ), and potentially down to better than $\sim 50 \mathrm{~nm}$. In this paper, firstly the proposed method was extensively investigated using a FEM tool. Secondly, an experimental validation was carried out using sample of known non-equi-biaxial RS depth profile. Besides proving the effectiveness of the method, these analyses highlighted conditions under which 
high accuracy is ensured. Indeed, correct selection of the main geometric dimension (i.e. ring-core diameter) accounting for the specific case study is of utmost importance for the success of the RS evaluation. This is undoubtedly a significant advancement in the field of RS assessment, with emphasis on nano- and micronscale coatings and treated surfaces.

Moreover, such a calculation approach is not only applicable to the sole ring-core geometry at the (sub)micron-scale, but it can also be potentially developed for other geometries and across the length-scales.

\section{Acknowledgements}

The authors would like to acknowledge Eric Le Bourhis (Universite de Poitiers) for providing the multilayered coating samples.

\section{References}

[1] Withers PJ, Bhadeshia HKDH. Residual stress. Part 2 - Nature and origins. Materials Science and Technology. 2001;17:366-75.

[2] James MN. Residual stress influences on structural reliability. Engineering Failure Analysis. 2011;18:1909-20.

[3] Salvati E, Sui T, Zhang H, Lunt AJG, Fong KS, Song X, et al. Elucidating the Mechanism of Fatigue Crack Acceleration Following the Occurrence of an Underload. Advanced Engineering Materials. 2016;18:2076-87.

[4] Salvati E, Zhang H, Fong KS, Song X, Korsunsky AM. Separating plasticity-induced closure and residual stress contributions to fatigue crack retardation following an overload. Journal of the Mechanics and Physics of Solids. 2017;98:222-35.

[5] Pintschovius L, Jung V, Macherauch E, Vöhringer O. Residual stress measurements by means of neutron diffraction. Materials Science and Engineering. 1983;61:43-50.

[6] Salvati E, Korsunsky AM. An analysis of macro- and micro-scale residual stresses of Type I, II and III using FIB-DIC micro-ring-core milling and crystal plasticity FE modelling. International Journal of Plasticity. 2017;98:123-38.

[7] Everaerts J, Salvati E, Uzun F, Romano Brandt L, Zhang H, Korsunsky AM. Separating macro- (Type I) and micro- (Type II+III) residual stresses by ring-core FIB-DIC milling and eigenstrain modelling of a plastically bent titanium alloy bar. Acta Materialia. 2018;156:43-51.

[8] Shiari B, Miller RE. Multiscale modeling of crack initiation and propagation at the nanoscale. Journal of the Mechanics and Physics of Solids. 2016;88:35-49.

[9] Holmberg K, Ronkainen H, Laukkanen A, Wallin K, Hogmark S, Jacobson S, et al. Residual stresses in TiN, DLC and MoS2 coated surfaces with regard to their tribological fracture behaviour. Wear. 2009;267:2142-56.

[10] Renzelli M, Mughal MZ, Sebastiani M, Bemporad E. Design, fabrication and characterization of multilayer CrCrN thin coatings with tailored residual stress profiles. Materials \& Design. 2016;112:162-71.

[11] Vierneusel B, Benker L, Tremmel S, Göken M, Merle B. Isolating the effect of residual stresses on coating wear by a mechanical stress relaxation technique. Thin Solid Films. 2017;638:159-66.

[12] Sumigawa T, Shishido T, Murakami T, Kitamura T. Interface crack initiation due to nano-scale stress concentration. Materials Science and Engineering: A. 2010;527:4796-803.

[13] Uzun F, Korsunsky AM. On the identification of eigenstrain sources of welding residual stress in bead-on-plate inconel 740H specimens. International Journal of Mechanical Sciences. 2018;145:231-45.

[14] DeWald AT, Hill MR. Eigenstrain-based model for prediction of laser peening residual stresses in arbitrary three-dimensional bodies Part 1: Model description. The Journal of Strain Analysis for Engineering Design. 2009;44:1-11.

[15] Puzikov VM, Tkachenko VF, Tsurikov VA. XRD method for the determination of internal stresses in KDP crystals and their relationship to the anomalous biaxiality. Functional Materials. 2015;22:402-7.

[16] Dufrenoy S, Chauveau T, Brenner R, Fontugne C, Bacroix B. Modeling methodology for stress determination by XRD in polycrystalline materials. Advanced Materials Research2014. p. 106-11.

[17] Holmberg J, Steuwer A, Stormvinter A, Kristoffersen H, Haakanen M, Berglund J. Residual stress state in an induction hardened steel bar determined by synchrotron- and neutron diffraction compared to results from labXRD. Materials Science and Engineering A. 2016;667:199-207.

[18] Nobre JP, Kornmeier M, Scholtes B. Plasticity Effects in the Hole-Drilling Residual Stress Measurement in Peened Surfaces. Experimental Mechanics. 2017;58:369-80.

[19] Schuster S, Steinzig M, Gibmeier J. Incremental Hole Drilling for Residual Stress Analysis of Thin Walled Components with Regard to Plasticity Effects. Experimental Mechanics. 2017;57:1457-67.

[20] Olson MD, Hill MR. Two-Dimensional Mapping of In-plane Residual Stress with Slitting. Experimental Mechanics. 2018;58:151-66.

[21] Pan Q, Mi Y, Wei Y, Ren Y. A method of testing residual stress by ultrasonic shear and longitudinal waves. 2016 IEEE International Conference on Mechatronics and Automation, IEEE ICMA 20162016. p. 1295-9. 
[22] Abadias G, Chason E, Keckes J, Sebastiani M, Thompson GB, Barthel E, et al. Review Article: Stress in thin films and coatings: Current status, challenges, and prospects. Journal of Vacuum Science and Technology A: Vacuum, Surfaces and Films. 2018;36.

[23] Kubec A, Niese S, Rosenthal M, Gluch J, Burghammer M, Gawlitza P, et al. Sub $25 \mathrm{~nm}$ focusing with a long working distance using multilayer Laue lenses. Journal of Instrumentation. 2018;13.

[24] Keckes J, Daniel R, Todt J, Zalesak J, Sartory B, Braun S, et al. $30 \mathrm{~nm}$ X-ray focusing correlates oscillatory stress, texture and structural defect gradients across multilayered TiN-SiOx thin film. Acta Materialia. 2018;144:86273.

[25] Nguyen TN, Nguyen VD, Jung S, Yi J. Raman scattering analysis of the residual stress in metal-induced crystallized amorphous silicon thin films using nickel. Applied Surface Science. 2009;255:8252-6.

[26] Zhu W, Marin E, Sugano N, Pezzotti G. Tensor-resolved Raman spectroscopic analysis of wear-induced residual stress fields in long-term alumina hip-joint retrievals. Journal of the Mechanical Behavior of Biomedical Materials. 2017;66:201-10.

[27] Guo Y, Abdolvand H, Britton TB, Wilkinson AJ. Growth of \{\} twins in titanium: A combined experimental and modelling investigation of the local state of deformation. Acta Materialia. 2017;126:221-35.

[28] Kartal ME, Kiwanuka R, Dunne FPE. Determination of sub-surface stresses at inclusions in single crystal superalloy using HR-EBSD, crystal plasticity and inverse eigenstrain analysis. International Journal of Solids and Structures. 2015;67-68:27-39.

[29] Wan VVC, Cuddihy MA, Jiang J, Maclachlan DW, Dunne FPE. An HR-EBSD and computational crystal plasticity investigation of microstructural stress distributions and fatigue hotspots in polycrystalline copper. Acta Materialia. 2016;115:45-57.

[30] Jiang J, Britton TB, Wilkinson AJ. The orientation and strain dependence of dislocation structure evolution in monotonically deformed polycrystalline copper. International Journal of Plasticity. 2015;69:102-17.

[31] Wolf H, Bohm W. The Ring-Core Method for Measuring Residual Stresses and its Use with Turbines and Generator Shafts. Archiv Eisenhiittenwesen. 1971;41:5.

[32] Ajovalasit A, Petrucci G, Zuccarello B. Determination of nonuniform residual stresses using the ring-core method. Journal of Engineering Materials and Technology, Transactions of the ASME. 1996;118:224-8.

[33] Schajer GS. Measurement of non-uniform residual stresses using the hole drilling method - part I: Stress calculation procedure. American Society of Mechanical Engineers, Materials Division (Publication) MD1988. p. 8591.

[34] Winiarski B, Benedetti M, Fontanari V, Allahkarami M, Hanan JC, Withers PJ. High Spatial Resolution Evaluation of Residual Stresses in Shot Peened Specimens Containing Sharp and Blunt Notches by Micro-hole Drilling, Micro-slot Cutting and Micro-X-ray Diffraction Methods. Experimental Mechanics. 2016;56:1449-63. [35] Zhu R, Xie H, Dai X, Zhu J, Jin A. Residual stress measurement in thin films using a slitting method with geometric phase analysis under a dual beam (FIB/SEM) system. Measurement Science and Technology. 2014;25. [36] Krottenthaler M, Benker L, Mughal MZ, Sebastiani M, Durst K, Göken M. Effect of elastic anisotropy on strain relief and residual stress determination in cubic systems by FIB-DIC experiments. Materials \& Design. 2016;112:505-11.

[37] Sasangka WA, Gan CL, Lai D, Tan CS, Thompson CV. Characterization of the Young's modulus, residual stress and fracture strength of $\mathrm{Cu}-\mathrm{Sn}$-In thin films using combinatorial deposition and micro-cantilevers. Journal of Micromechanics and Microengineering. 2015;25.

[38] Korsunsky AM, Sebastiani M, Bemporad E. Focused ion beam ring drilling for residual stress evaluation. Materials Letters. 2009;63:1961-3.

[39] Korsunsky AM, Sebastiani M, Bemporad E. Residual stress evaluation at the micrometer scale: Analysis of thin coatings by FIB milling and digital image correlation. Surface and Coatings Technology. 2010;205:2393-403.

[40] Sebastiani M, Bemporad E, Carassiti F, Schwarzer N. Residual stress measurement at the micrometer scale: Focused ion beam (FIB) milling and nanoindentation testing. Philosophical Magazine. 2011;91:1121-36.

[41] Menda F, Šarga P, Trebuňa F. Estimation of residual stress field uniformity when using the ring-core method. Advanced Materials Research2014. p. 325-30.

[42] Zhu JG, Xie HM, Li YJ, Hu ZX, Luo Q, Gu CZ. Interfacial Residual Stress Analysis of Thermal Spray Coatings by Miniature Ring-Core Cutting Combined with DIC Method. Experimental Mechanics. 2014;54:127-36.

[43] Lunt AJG, Salvati E, Ma L, Dolbyna IP, Neo TK, Korsunsky AM. Full in-plane strain tensor analysis using the microscale ring-core FIB milling and DIC approach. Journal of the Mechanics and Physics of Solids. 2016;94:47-67. [44] Lunt AJG, Baimpas N, Salvati E, Dolbnya IP, Sui T, Ying S, et al. A state-of-the-art review of micron-scale spatially resolved residual stress analysis by FIB-DIC ring-core milling and other techniques. The Journal of Strain Analysis for Engineering Design. 2015;50:426-44.

[45] Salvati E, Sui T, Lunt AJG, Korsunsky AM. The effect of eigenstrain induced by ion beam damage on the apparent strain relief in FIB-DIC residual stress evaluation. Materials \& Design. 2016;92:649-58.

[46] Salvati E, Brandt LR, Papadaki C, Zhang H, Mousavi SM, Wermeille D, et al. Nanoscale structural damage due to focused ion beam milling of silicon with Ga ions. Materials Letters. 2018;213:346-9.

[47] Salvati E, Sui T, Korsunsky AM. Uncertainty quantification of residual stress evaluation by the FIB-DIC ringcore method due to elastic anisotropy effects. International Journal of Solids and Structures. 2016;87:61-9. 
[48] Korsunsky AM, Salvati E, Lunt AGJ, Sui T, Mughal MZ, Daniel R, et al. Nanoscale residual stress depth profiling by Focused Ion Beam milling and eigenstrain analysis. Materials \& Design.

[49] Schajer GS, Rickert TJ. Incremental Computation Technique for Residual Stress Calculations Using the Integral Method. Experimental Mechanics. 2011;51:1217-22.

[50] Zuccarello B. Optimal calculation steps for the evaluation of residual stress by the incremental hole-drilling method. Experimental Mechanics. 1999;39:117-24.

[51] Zuccarello B. Optimization of depth increment distribution in the ring-core method. Journal of Strain Analysis for Engineering Design. 1996;31:251-8.

[52] Kinoshita N, Mura T. EIGENSTRAIN PROBLEMS IN A FINITE ELASTIC BODY. SIAM Journal on Applied Mathematics. 1984;44:524-35.

[53] Korsunsky AM. A Teaching Essay on Residual Stresses and Eigenstrains2017.

[54] Korsunsky AM, Guénolé J, Salvati E, Sui T, Mousavi M, Prakash A, et al. Quantifying eigenstrain distributions induced by focused ion beam damage in silicon. Materials Letters. 2016;185:47-9.

[55] Romano-Brandt L, Salvati E, Le Bourhis E, Moxham T, Dolbnya I, Korsunsky AM. The Effect of Magnetron Sputtering Pressure on the Nano-Scale Depth Profiles of Residual Stress in Cu/W Nano-Multilayer Coatings. Submitted for publication (under review). 2019.

[56] Romano Brandt L, Salvati E, Papadaki C, Zhang H, Ying S, Le Bourhis E, et al. Probing the deformation and fracture properties of $\mathrm{Cu} / \mathrm{W}$ nano-multilayers by in situ SEM and synchrotron XRD strain microscopy. Surface and Coatings Technology. 2017;320:158-67.

[57] Senn M. Digital Image Correlation and Tracking. [Computer Software].

2015; https://uk.mathworks.com/matlabcentral/ fileexchange/50994-digital-image-correlation-and-tracking. 